https://doi.org/10.7559/gestaoedesenvolvimento.2019.379

Data de receção: 13/03/2019 Data de aceitação: 01/07/2019

\title{
ADAPTAÇÃO CULTURAL, VALIDADE E FIABILIDADE DA ESCALA PESSOAL DE RESULTADOS-CRIANÇAS E JOVENS
}

\section{CULTURAL ADAPTATION, VALIDITY AND RELIABILITY OF SCALE OF CHILDREN AND YOUTH PERSONAL RESULTS}

\author{
Cristina Simões ${ }^{1}$ orcid.org/0000-0002-5136-255X \\ Célia Ribeiro $^{2}$ orcid.org/0000-0002-1000-6890 \\ Inês Cabral $^{3}$
}

Paulo Almeida Pereira ${ }^{4}$ orcid.org/0000-0002-3941-8274

\begin{abstract}
Resumo: A presente investigação apresenta os resultados de um estudo piloto que tem como objetivos efetuar a adaptação cultural da Escala Pessoal de Resultados-Crianças e Jovens (EPR-CJ), bem como examinar a sua validade e fiabilidade. Esta escala possibilita avaliar a Qualidade de Vida $(Q V)$ das crianças e jovens com Dificuldade Intelectual (DI). Das diferentes amostras englobadas nesta investigação, salienta-se que a amostra final incluiu 54 crianças $e$ jovens com DI e 54 profissionais de educação. O estudo iniciou com a aplicação das diretrizes internacionais relativas à adaptação cultural. Os resultados do Índice de Validade de Conteúdo (IVC) dos itens, do acordo universal do IVC do instrumento, da média do IVC da escala e dos valores do Kappa de Cohen suportaram a validade de conteúdo, tendo as correlações de Pearson reforçado a validade de constructo da EPR-CJ. Os valores do teste-reteste, da consistência interna e do
\end{abstract}

\footnotetext{
${ }^{1}$ Doutorada em Ciências da Educação, Área de Especialização em Educação Especial. Docente de Educação Especial. E-mail: cristinasimoes7@ sapo.pt

2 Doutorada em Psicologia, Área de Especialização em Psicologia Pedagógica. Professora Auxiliar da Universidade Católica Portuguesa -Viseu. E-mail: cribeiro@viseu.ucp.pt

${ }^{3}$ Mestre em Ciências da Educação, Especialização em Educação Especial. Docente de Educação Especial. E-mail: minesleiria@ hotmail.com

${ }^{4}$ Professor Auxiliar da Universidade Católica Portuguesa - Viseu - Instituto de Gestão e das Organizações da Saúde. E-mail: ppereira@ viseu.ucp.pt
} 
acordo entre observadores sustentaram a fiabilidade do instrumento. Os diferentes coeficientes psicométricos ilustraram que esta escala pode ser bastante útil para a avaliação da $Q V$ das crianças e jovens com DI, sendo a EPR-CJ crucial para desenhar os programas de intervenção destes alunos em contextos inclusivos de aprendizagem.

Palavras-chave: Dificuldade Intelectual, Qualidade de Vida, Validade, Fiabilidade.

\begin{abstract}
The present research presents the results of an initial study that aims to analyze the cross-cultural adaptation of the Personal Outcomes Scale-Children and Adolescents (POS-C), as well as to examine its validity and reliability. This scale assesses the Quality of Life (QOL) of children and adolescents with Intellectual Disability (ID). Of the several samples involved in the research, it is stressed that the final sample included 54 children and adolescents with ID and 54 education support staff. The research started with the application of the cross-cultural adaptation' guidelines. The results of the Content Validity Index (CVI) of each item, the scale CVI-universal agreement, the scale CVI average and the Cohen's kappa scores supported the content validity, and the Pearson's correlations confirmed the construct validity of the POS-C. The test-retest, the internal consistency and the inter-rater reliability supported the reliability of the tool. The different psychometric coefficients demonstrate that this scale may be advantageous to assess the QOL of children and adolescents with ID, as well as to design the intervention programs of those students in inclusive contexts of learning.
\end{abstract}

Keywords: Intellectual Disability; Quality of Life; Validity; Reliability.

\title{
INTRODUÇÃO
}

O conceito de Qualidade de Vida (QV) tem vindo a assumir, paulatinamente, um papel relevante na vida de todos os cidadãos e, a partir da década oitenta (Schalock \& Verdugo, 2002), começou a ser ventilado na área da Dificuldade Intelectual (DI). A defesa pela igualdade de 
oportunidades e equidade entre todas as pessoas impulsionou o interesse progressivo pelo estudo da QV da população com DI, ao longo das suas várias etapas de vida (Schalock, Gardner, \& Bradley, 2007).

A QV inclui as noções de autodeterminação, inclusão, capacitação e igualdade entre todos (Schalock \& Verdugo, 2002), proporcionando uma linguagem comum e universal capaz de dimensionar as pessoas com deficiência num quadro social e ecológico (Simões, Santos, Biscaia, \& Thompson, 2016), que combate a discriminação e refresca a noção da capacitação das pessoas com DI.

Assim, a presente investigação pretende colmatar o vazio existente na literatura sobre a QV de crianças e jovens com DI que frequentam a escolaridade obrigatória, sendo necessário e urgente desenvolverem-se instrumentos de avaliação da QV nesta faixa etária (Ghotra, McIsaac, Kirk, \& Kuhle, 2016; Huang, Wang, Tang, Chen, \& Yu, 2017). Deste modo, este estudo tem como objetivos: (a) efetuar a adaptação cultural da Escala Pessoal de Resultados-Crianças e Jovens (EPR-CJ); (b) examinar a validade da EPR-CJ, através da validade de conteúdo e da validade de constructo; e (c) analisar a fiabilidade da EPR-CJ, com base no testereteste, na consistência interna e no acordo entre observadores.

\section{DEFINIÇÃO DE DIFICULDADE INTELECTUAL}

A terminologia de DI tem sofrido diversas modificações ao longo do tempo, que refletem o esforço que se tem verificado nesta área para se adotar uma linguagem mais atualizada e menos ofensiva para estas pessoas. Em 2007, verificou-se uma substituição do termo 'deficiência mental' por 'DI' pela prestigiada American Association on Intellectual and Developmental Disabilities (AAIDD; Schalock et al., 2007).

Schalock e colaboradores (2007) esclarecem que enquanto o anterior conceito destacava as limitações no funcionamento individual, a nomenclatura 'DI' tem como ponto fulcral a interação entre o indivíduo e os seus contextos individuais, reconhecendo a importância dos apoios para melhorar a funcionalidade e, naturalmente, a QV. Segundo Morato e Santos (2007), a utilização do termo 'dificuldade' torna-se mais adequado por ser menos estigmatizante, preconizando uma expetativa mais positiva quando comparada com o termo 'deficiência', ao mesmo tempo que 
subjaz a eliminação da perspetiva psicométrica, onde o enfoque se circunscreve à pessoa.

Para a AAIDD, a partir de 2002, a DI é entendida como um funcionamento geral significativamente abaixo da média, onde as limitações (dois desvios-padrão abaixo da média) do funcionamento intelectual em concomitância com as limitações adaptativas (dois desviospadrão abaixo da média) se traduzem nas habilidades concetuais, práticas e sociais, sendo os 18 anos a idade para o diagnóstico desta condição (Luckasson et al., 2002; Schalock et al., 2007). Segundo a American Psychiatric Association (2014), a DI pressupõe: alterações no QI (raciocínio, resolução de problemas, planificação, pensamento abstrato, julgamento, aprendizagem académica e aprendizagem prática); alterações no comportamento adaptativo, que interferem no cumprimento das normas socioculturais e do desenvolvimento necessárias para a autonomia pessoal e a responsabilidade social; e deve ter início durante o período de desenvolvimento humano.

Genericamente, observa-se uma congruência nos critérios de diagnóstico entre estes dois organismos internacionais (Schalock et al., 2007). A definição de DI não considera somente o QI, reforçando a funcionalidade da pessoa nos seus envolvimentos de vida (Morato \& Santos, 2007). Os apoios são mediadores fundamentais entre o nível de funcionalidade atual (presente) e o nível de funcionalidade que cada indivíduo com DI pode vir a ter (desejado/optimal), sublinhando-se que são preditores da QV da população com DI (Lombardi, Croce, Claes, Vandevelde, \& Schalock, 2016; Simões et al., 2016). Por conseguinte, a definição de DI é mais abrangente do que uma condição biomédica ou um défice psicológico e educativo, abrangendo a interação entre o indivíduo e os seus contextos de vida, bem como os direitos e papéis exercidos nas suas comunidades (Schalock, Luckasson, Tassé, \& Verdugo, 2018). 


\section{QUALIDADE DE VIDA E DIFICULDADE INTELECTUAL}

A QV tem sido considerada fundamental para a prestação dos apoios personalizados que almejam melhores resultados pessoais (Buntinx \& Schalock, 2010), para a planificação centrada na pessoa (Claes, Van Hove, Vandevelde, Van Loon, \& Schalock, 2010a) e para orientar os serviços e as políticas (Brown, 2017; Buntinx \& Schalock, 2010), assentando nas práticas baseadas em evidências (Schalock, Verdugo, \& Gómez, 2011) que sustentam a qualidade da intervenção efetuada com esta população. Assim, o quadro concetual da QV tornou-se imperativo para uma melhor prestação de serviços à população com DI, potenciando uma maior compreensão do estilo de vida de cada pessoa (Simões et al., 2016) e o conhecimento do impacto das características pessoais e contextuais (Simões \& Santos, 2017) no seu percurso de vida.

Evidentemente que o tema da QV não é linear, na medida em que na literatura existem diversas correntes para suportar este constructo e, de acordo com Schalock e colaboradores (2002) existem mais de 100 definições associadas a este conceito. A primeira dificuldade prende-se na confusão que circunda o conceito, muitas vezes sendo-lhe atribuído o sinónimo de bem-estar, satisfação, felicidade ou QV relacionada com a saúde (Oliveira, Ribeiro, Simões, \& Pereira, 2018). Enfatiza-se que a panóplia de definições existentes reflete as perspetivas dos investigadores de diversas áreas, nem sempre havendo uma fundamentação do constructo com base em estudos psicométricos (Li, Tsoi, Zhang, Chen, \& Wang, 2013).

Atentos a todos estes obstáculos, um painel de especialistas pronunciou-se sobre os princípios que devem guiar a definição da QV na população com DI (Schalock et al., 2002). Respeitando todas as diretrizes internacionalmente estipuladas, surgiu o modelo de Schalock e Verdugo (2002), que pretendeu que a conceção da QV na DI passasse de uma noção abstrata para um constructo empiricamente mensurável.

A definição de QV é, então, caracterizada pelos seguintes princípios: é um constructo multidimensional, influenciado por fatores pessoais e envolvimentais; é composto por indicadores subjetivos e objetivos, contudo é a perceção do indivíduo que reflete a QV que o próprio experimenta; compreende os mesmos domínios de vida que são 
importantes para todos os cidadãos; a QV existe quando as necessidades específicas são resolvidas e cada aluno tem oportunidade para participar nas atividades da sua comunidade e enriquecer as suas experiências de vida; e envolve as escolhas, necessidades e preferências pessoais (Schalock et al., 2002; Schalock \& Verdugo, 2002). De um modo mas sintético, pode-se definir a QV como

\begin{abstract}
um fenómeno multidimensional composto por domínios centrais que constituem o bem-estar pessoal. Estes domínios são influenciados por características pessoais e fatores contextuais. A qualidade de vida é o produto destes fatores e pode ser positivamente impactada através de estratégias de melhoria da qualidade que englobam o desenvolvimento de talentos pessoais, maximizando o envolvimento pessoal, fornecendo apoios individualizados e facilitando as oportunidades de crescimento pessoal (Schalock, Verdugo, Gómez, \& Reinders, 2016, p. 2).
\end{abstract}

A universalidade do conceito, olhando para todos os cidadãos de igual forma, levou a que os autores definissem oito domínios centrais de vida, nomeadamente: Desenvolvimento Pessoal, Autodeterminação, Relações Interpessoais, Inclusão Social, Direitos, Bem-Estar Emocional, BemEstar Físico e Bem-Estar Material (Schalock \& Verdugo, 2002). Salientase que este modelo tem vindo a ser alvo de estudos em diversos países, sendo na opinião de Gómez, Verdugo, Arias, e Arias (2011) o constructo de QV mais investigado e implementado na área da DI. Concomitantemente, um estudo comparativo entre modelos de QV conferiu uma maior adequação do constructo de Schalock e Verdugo (2002) para ser aplicado na avaliação da QV na população com DI (Simões, Santos, \& Claes, 2015a).

Não obstante o constructo da QV na DI ter começado a ser investigado em Portugal (Simões, Santos, \& Biscaia, 2016; Simões, Santos, Claes, Van Loon, \& Schalock, 2017), os investigadores privilegiaram a idade adulta. Assim, os estudos realizados sobre a QV de crianças e jovens em contexto escolar são escassos (Gaspar \& Matos, 2008; Oliveira et al., 2018), tendo sido a sua maioria efetuados na área da pediatria (Araújo, Dourado, \& Ferreira, 2015). Apesar de existirem instrumentos validados 
para crianças e adolescentes (Araújo et al., 2015; Gaspar \& Matos, 2008) e para a população adulta com DI (Simões et al., 2017), não existe, até ao momento, uma escala que possibilite avaliar a QV de crianças e jovens com DI.

Embora este modelo tenha sido, inicialmente, aplicado à idade adulta, advoga-se a sua importância para possibilitar repensar-se a intervenção efetuada ao longo da escolaridade, que deve ocorrer na escola inclusiva (Claes, Mostert, Moonen, Van Loon, \& Schalock, 2015; Gaspar \& Matos, 2008; Oliveira et al., 2018). Simões e Santos (2018) reforçam que os apoios promotores da inclusão devem basear-se numa metodologia multidimensional, onde os indicadores de QV assumem um papel preponderante na implementação dos programas educativos individuais, assim como na avaliação dos resultados pessoais obtidos e na eficácia das práticas implementadas. Os apoios oferecidos pela escola são um componente fundamental para promoverem uma vida adulta com mais qualidade (Oliveira et al., 2018), devendo estimular a aprendizagem de competências referentes aos oito domínios de QV (Schalock \& Verdugo, 2002; Simões \& Santos, 2018).

No entanto, em Portugal, não existem escalas que permitam avaliar a QV das crianças e dos jovens com DI. Os seus currículos parecem, frequentemente, ainda estarem mais focados nas atividades de lazer (Simões \& Santos, 2017), sem estimularem outras facetas de vida conducentes à independência, à participação social e ao bem-estar (Simões et al., 2017). Por conseguinte, é importante adaptar-se e validarse uma escala que possibilite medir a QV nesta faixa etária, contribuindo para se repensar a intervenção educativa potenciadora da inclusão.

\section{METODOLOGIA}

\subsection{Caraterização da Amostra}

Utilizou-se o método de amostragem não probabilístico por conveniência, uma vez que os participantes foram selecionados de acordo com os seguintes critérios: serem crianças ou jovens com DI, terem idades compreendidas entre os 10 e os 17 anos e 11 meses e residir na zona centro do país. Assim, a amostra é constituída por 54 crianças e jovens com DI e 
pelos respetivos cuidadores que conheciam cada participante há pelo menos dois anos.

A Tabela 1 apresenta os dados de caraterização das crianças e jovens com DI, dos quais 29 são do género masculino e 25 do género feminino. A idade destes participantes estava compreendida entre os 10 e os 17 anos (Midade $=12.48, D P=2.93$ ). Estas crianças e jovens apresentavam um diagnóstico de DI ligeira ( $n=22,40.74 \%)$, moderada $(n=17,31.48 \%)$ e grave $(n=15,27.78 \%)$. Oito dos participantes $(14.81 \%)$ eram formandos da formação profissional, enquanto os restantes ( $n=46,85.19 \%)$ frequentavam a escolaridade obrigatória.

Tabela 1 Caraterização das crianças e jovens

\begin{tabular}{lccc}
\hline & & $n$ & $\%$ \\
\hline Género & Masculino & 29 & 53.70 \\
& Feminino & 25 & 46.30 \\
\hline Diagnóstico & DI ligeira & 22 & 40.74 \\
& DI moderada & 17 & 31.48 \\
& DI grave & 15 & 27.78 \\
\hline Idade & $10-12$ & 27 & 50.00 \\
& $13-17$ & 27 & 50.00 \\
\hline Habilitações literárias & $1 .^{\circ}$ ciclo & 12 & 22.22 \\
& $2 .^{\circ}$ ciclo & 23 & 42.59 \\
& $3 .^{\circ}$ ciclo & 16 & 29.63 \\
& Ensino secundário & 3 & 5.56 \\
\hline Atividade & Estudante & 46 & 85.19 \\
& Formando & 8 & 14.81 \\
\hline
\end{tabular}

Relativamente à caraterização dos cuidadores (Tabela 2), verifica-se que a totalidade da segunda parte da EPR-CJ foi preenchida por profissionais de educação do género feminino $(n=54,100 \%)$. Estes eram professores $(n=37,68.50 \%)$ e psicólogos $(n=17,31.50 \%)$ que conheciam bem cada participante com DI. A idade destes cuidadores estava compreendida entre os 31 e os 60 anos $\left(M_{\text {idade }}=46.59, D P=5.68\right)$, sendo que a maioria tinha como habilitações literárias a licenciatura $(n=33$, $61.10 \%)$ 
Tabela 2 Caraterização dos cuidadores

\begin{tabular}{lccc}
\hline & & $n$ & $\%$ \\
\hline Género & Masculino & 0 & 0 \\
& Feminino & 54 & 100 \\
\hline Idade & $31-40$ & 9 & 16.67 \\
& $41-50$ & 28 & 51.85 \\
& $51-60$ & 17 & 31.48 \\
\hline Habilitações literárias & Bacharelato & 3 & 5.56 \\
& Licenciatura & 33 & 61.10 \\
& Mestrado & 15 & 27.78 \\
& Doutoramento & 3 & 5.56 \\
\hline Relação com os alunos & Professores & 37 & 68.50 \\
& Psicólogos & 17 & 31.50 \\
\hline
\end{tabular}

\subsection{Instrumento}

Na presente investigação foi utilizada a EPR-CJ. Esta escala é a versão portuguesa da Personal Outcomes Scale-Children and Adolescents (POSC; Claes et al., 2015), permitindo avaliar a QV de crianças com idades compreendidas entre os 6 e os 17 anos e 11 meses. Salienta-se que, em Portugal, a escala já foi validada para pessoas adultas com DI, tendo apresentado características psicométricas robustas (Simões \& Santos, 2014; Simões, Santos, \& Claes, 2015b; Simões et al., 2017).

A EPR-CJ mede a QV baseada nos oito domínios de Schalock e Verdugo (2002). A escala também possibilita avaliar três fatores de QV: Independência, que engloba os domínios do Desenvolvimento Pessoal e Autodeterminação; Participação Social, que abarca os domínios das Relações Interpessoais, Inclusão Social e Direitos; e Bem-Estar, que abrange os domínios do Bem-Estar Emocional, Bem-Estar Físico e BemEstar Material (Simões et al., 2017).

$\mathrm{O}$ instrumento de avaliação da QV encontra-se dividido em duas secções, uma para crianças e jovens (autorrelato) e outra para pais (relato dos cuidadores). As duas partes da EPR-CJ apresentam o mesmo número de perguntas (seis por domínio). A diferença entre ambas as secções encontra-se na pessoa que avalia a $\mathrm{QV}$ e na linguagem utilizada para formular as perguntas (Claes et al., 2015).

A escala tem um total de quarenta e oito itens em cada parte, medidos através de uma escala com formato Likert de três pontos ( $3=$ frequentemente, $2=$ =às vezes, $1=$ nunca). Os valores da escala podem ser 
interpretados em função dos domínios, dos fatores e do total de QV (Claes et al., 2015).

\subsection{Procedimentos}

A presente investigação baseou-se numa abordagem quantitativa, descritiva, correlacional e transversal. Foram elaborados três documentos intitulados Informação ao Participante, sendo um destinado aos Professores/Diretores de Turma, outro aos Pais/Encarregados de Educação e outro aos Diretores dos Agrupamentos de Escolas. Os referidos documentos foram acompanhados por uma Declaração de Consentimento, tendo por base a Declaração de Helsínquia (World Medical Association, 2008).

A aplicação da EPR-CJ decorreu ao longo do segundo semestre de 2016, tendo sido fundamental, a colaboração dos docentes de Educação Especial no estabelecimento do primeiro contacto com os alunos e respetivos pais/encarregados de educação, assim como na organização do local onde decorreu a aplicação. $\mathrm{O}$ instrumento foi aplicado de acordo com as diretrizes da escala original (Claes et al., 2015), sendo a parte do autorrelato sempre aplicada através de uma entrevista pelas autoras da investigação. As duas partes da EPR-CJ foram respondidas de forma independente pelos diferentes participantes.

Para o tratamento estatístico dos dados utilizou-se o programa Microsoft Excel e o programa Statistical Package for the Social Sciences (SPSS), versão 21.0.

\section{APRESENTAÇÃO DOS RESULTADOS}

\subsection{Adaptação Cultural e Validade de Conteúdo}

A investigação começou com a adaptação cultural do instrumento, respeitando-se as fases descritas na literatura (Simões \& Santos, 2014). Assim, iniciou-se pela fase da tradução, que envolveu dois tradutores com conhecimento da língua inglesa. As duas versões foram comparadas, não tendo sido observadas diferenças entre os tradutores que participaram neste processo. Seguiu-se a fase da retro-tradução, que evolveu dois tradutores cuja língua materna era o inglês. A comparação destas versões confirmou a versão da escala inicialmente traduzida. 
Após a fase da adaptação cultural, procedeu-se à validade de conteúdo empírica do instrumento, com a avaliação quantitativa da EPR-CJ por 10 especialistas na área da DI, conforme é recomendado na literatura (Polit \& Beck, 2006; Simões \& Santos, 2014; Yaghmaie, 2003). Os especialistas avaliaram, através de uma escala Likert de 4 opções (Wynd, Schmidt, \& Schaefer, 2003; Yaghmaie, 2003), a relevância, a clareza, a simplicidade e a ambiguidade de cada item do instrumento (Yaghmaie, 2003). Estas 4 opções foram transformadas numa escala dicotómica, sendo que as respostas 1 e 2 (irrelevante e pouco relevante) significaram não haver validade de conteúdo e as classificações 3 e 4 (relevante e muito relevante) indicaram haver validade de conteúdo (Polit \& Beck, 2006; Yaghmaie, 2003). Deste modo, procedeu-se ao cálculo da proporção de acordo entre os especialistas, ao Índice de Validade de Conteúdo (IVC) de cada item, ao acordo universal do IVC da escala, à média do IVC da escala e ao kappa de Cohen.

Conforme se observa na Tabela 3, a proporção de acordo entre os 10 especialistas que participaram na validade de conteúdo da EPR-CJ variou entre .95 e 1.00. Na relevância, só o perito 4 apresentou um acordo de .99 , enquanto os restantes consideraram que todos os itens da EPR-CJ eram relevantes. Relativamente à clareza e à simplicidade das perguntas, os valores entre os especialistas oscilaram entre .96 e 1.00 , enquanto que na ambiguidade variaram entre .95 e .99 .

Tabela 3 Proporção de acordo entre os peritos

\begin{tabular}{l|c|c|c|c}
\hline & Relevância & Clareza & Simplicidade & Ambiguidade \\
\cline { 2 - 5 } Perito 1 & 1.00 & .96 & .96 & .97 \\
\hline Perito 2 & 1.00 & 1.00 & 1.00 & .99 \\
\hline Perito 3 & 1.00 & 1.00 & .99 & .97 \\
\hline Perito 4 & .99 & 1.00 & .99 & .95 \\
\hline Perito 5 & 1.00 & .98 & .99 & .98 \\
\hline Perito 6 & 1.00 & 1.00 & 1.00 & .98 \\
\hline Perito 7 & 1.00 & 1.00 & .99 & .99 \\
\hline Perito 8 & 1.00 & .99 & 1.00 & .95 \\
\hline Perito 9 & 1.00 & .96 & .97 & .97 \\
\hline Perito 10 & 1.00 & .98 & .99 & .99 \\
\hline
\end{tabular}


A proporção de acordo entre os especialistas pode mostrar valores inflacionados (Polit \& Beck, 2006; Watkins \& Pacheco, 2000; Wynd et al., 2003), pelo que os restantes índices refletem valores mais objetivos da validade de conteúdo. O IVC dos itens deve ser $\geq .78$ (Polit \& Beck, 2006), devendo ser retirados do instrumento os itens com valores inferiores a .75 (Yaghmaie, 2003). O IVC dos itens da EPR-CJ variou entre .80 e 1.00, não tendo sido observados valores abaixo de .75 .

A validade de conteúdo é assumida quando o acordo universal do IVC do instrumento é $\geq .80$ e a média do IVC da escala é $\geq .90$ (Polit \& Beck, 2006). Conforme a Tabela 4 mostra, o acordo universal do IVC da escala variou entre .84 (ambiguidade) e .99 (relevância). A média do IVC da EPR-CJ na relevância $(\mathrm{IVC}=1.00)$, na clareza $(\mathrm{IVC}=.99)$, na simplicidade (IVC=.99) e na ambiguidade (IVC=.97) também confirmou a validade de conteúdo do instrumento.

Tabela 4 Validade de conteúdo da Escala Pessoal de Resultados-

Crianças e Jovens

\begin{tabular}{l|c|c|c|c}
\hline & Relevância & Clareza & Simplicidade & Ambiguidade \\
\hline $\begin{array}{l}\text { Acordo universal } \\
\text { do IVC da escala }\end{array}$ & .99 & .90 & .91 & .84 \\
\hline $\begin{array}{l}\text { Média do IVC da } \\
\text { escala }\end{array}$ & 1.00 & .99 & .99 & .97 \\
\hline
\end{tabular}

Os valores do Kappa de Cohen abaixo de .40 refletem que existe um acordo fraco entre os especialistas, entre .41 e .60 o acordo é considerado moderado (Watkins \& Pacheco, 2000; Wynd et al., 2003), entre .61 e .75 os valores refletem um bom acordo e acima de .75 um excelente acordo (Watkins \& Pacheco, 2000). Conforme os dados descritos na Tabela 5, os valores do kappa de Cohen oscilaram entre .44 (acordo moderado) e .96 (acordo excelente). Observaram-se 9 acordos moderados e 8 acordos bons entre os pares de especialistas, sendo que a maioria dos valores deste IVC ilustrou um acordo excelente entre os peritos $(\mathrm{k}>.75)$. 
Tabela 5 Validade de conteúdo da escala (kappa de Cohen)

\begin{tabular}{l|c|c|c|c|c|c|c|c|c}
\hline & P1 & P2 & P3 & P4 & P5 & P6 & P7 & P8 & P9 \\
\hline P2 & .96 & & & & & & & & \\
\hline P3 & .73 & .87 & & & & & & & \\
\hline P4 & .84 & .89 & .74 & & & & & & \\
\hline P5 & .81 & .91 & .73 & .44 & & & & & \\
\hline P6 & .91 & .80 & .87 & .80 & .83 & & & & \\
\hline P7 & .91 & .80 & .87 & .80 & .83 & .50 & & & \\
\hline P8 & .76 & .92 & .45 & .56 & .62 & .85 & .85 & & \\
\hline P9 & .65 & .95 & .57 & .44 & .49 & .90 & .90 & .72 & \\
\hline P10 & .84 & .89 & .77 & .59 & .66 & .50 & .80 & .75 & .81 \\
\hline
\end{tabular}

\subsection{Validade de Constructo}

Para se examinar a validade de constructo da EPR-CJ, foram calculadas correlações de Pearson. As correlações de Pearson podem ser classificadas de moderadas ( $r=.40-.69)$, altas $(r=.70-.89)$ ou muito altas ( $r \geq .90$; Pestana \& Gageiro, 2005). A validade de constructo é alta quando o $r \geq .60$ entre pelo menos duas medidas ou o $r \geq .70$ numa medida (Hartley $\&$ MacLean, 2006).

Na Tabela 6 são apresentadas as correlações de Pearson relativamente aos domínios da primeira parte da EPR-CJ. Os valores dos domínios foram calculados através da soma dos respetivos indicadores de QV. No autorrelato, os dados mostram que se verificou pelo menos uma correlação moderada $(r=.40-.69)$ entre os domínios de QV, exceto no Bem-Estar Material. O valor mais elevado foi observado entre os domínios do Desenvolvimento Pessoal e da Inclusão Social ( $r=.63$ ). Não obstante, as correlações entre o Índice de QV do autorrelato e os oito domínios variaram entre valores moderados ( $r=.42$ no Bem-Estar Emocional) a altos ( $r=.82$ na Inclusão Social). 
Tabela 6 Intercorrelações entre os domínios (autorrelato)

\begin{tabular}{l|l|l|l|l|l|l|l|l}
\hline Domínios & DP & AD & RI & IS & D & BE & BF & BM \\
\hline $\mathrm{DP}$ & & & & & & & & \\
\hline $\mathrm{AD}$ & $.54^{* *}$ & & & & & & & \\
\hline $\mathrm{RI}$ & .05 & .13 & & & & & & \\
\hline $\mathrm{IS}$ & $.63^{* *}$ & $.49^{* *}$ & $.32^{*}$ & & & & & \\
\hline $\mathrm{D}$ & $.40^{* *}$ & $.52^{* *}$ & .24 & $.52^{* *}$ & & & & \\
\hline $\mathrm{BE}$ & .22 & .11 & $.53^{* *}$ & .16 & .13 & & & \\
\hline $\mathrm{BF}$ & .09 & .03 & .19 & $.38^{*}$ & $.44^{* *}$ & .12 & & \\
\hline $\mathrm{BM}$ & .21 & .23 & .20 & .04 & .28 & .12 & $.33^{*}$ & \\
\hline Indice de QV & $.57^{* *}$ & $.66^{* *}$ & $.54^{* *}$ & $.82^{* *}$ & $.71^{* *}$ & $.42^{* *}$ & $.55^{* *}$ & $.45^{* *}$ \\
\hline
\end{tabular}

Nota: $\quad \mathrm{DP}=$ Desenvolvimento $\quad$ Pessoal; $\quad \mathrm{AD}=$ Autodeterminação; $\quad \mathrm{RI}=$ Relações Interpessoais; IS=Inclusão Social; D=Direitos; $\mathrm{BE}=\mathrm{Bem}$-Estar Emocional; $\mathrm{BF}=\mathrm{Bem}$ Estar Físico; BM=Bem-Estar Material; QV=Qualidade de Vida; ${ }^{* *} p<.001 ;{ }^{*} p<.05$.

No relato dos cuidadores (Tabela 7), os dados mostram que se verificou pelo menos uma correlação moderada $(r=.40-.69)$ entre os oito domínios de QV. Tal como foi observado no autorrelato, a correlação mais elevada foi observada entre os domínios do Desenvolvimento Pessoal e da Inclusão Social ( $r=.65$ ). As correlações entre o Índice de QV do relato dos cuidadores e os oito domínios variaram entre valores moderados ( $r=.49$ no Bem-Estar Emocional) a altos ( $r=.85$ nas Relações Interpessoais).

Tabela 7 Intercorrelações entre os domínios (relato dos cuidadores)

\begin{tabular}{l|l|l|l|l|l|l|l|l}
\hline Domínios & DP & AD & RI & IS & D & BE & BF & BM \\
\hline DP & & & & & & & & \\
\hline AD & .25 & & & & & & & \\
\hline RI & $.38^{* *}$ & $.53^{* *}$ & & & & & & \\
\hline IS & $.65^{* *}$ & .18 & $.54^{* *}$ & & & & & \\
\hline D & $.57^{* *}$ & .11 & $.60^{* *}$ & $.49^{* *}$ & & & & \\
\hline $\mathrm{BE}$ & .08 & $.40^{* *}$ & $.48^{* *}$ & .04 & .26 & & & \\
\hline $\mathrm{BF}$ & $.30^{*}$ & .08 & $.37^{*}$ & $.37^{* *}$ & $.41^{* *}$ & .23 & & \\
\hline $\mathrm{BM}$ & .16 & .14 & $.42^{* *}$ & $.31^{*}$ & $.29^{*}$ & $.34^{*}$ & $.48^{* *}$ & \\
\hline $\begin{array}{l}\text { Índice de } \\
\text { QV }\end{array}$ & $.63^{* *}$ & $.52^{* *}$ & $.85^{* *}$ & $.69^{* *}$ & $.71^{* *}$ & $.49^{* *}$ & $.55^{* *}$ & $.63^{* *}$ \\
\hline
\end{tabular}

Nota: $\quad \mathrm{DP}=$ Desenvolvimento $\quad$ Pessoal; $\quad \mathrm{AD}=$ Autodeterminação; $\quad \mathrm{RI}=$ Relações Interpessoais; IS=Inclusão Social; $\mathrm{D}=\mathrm{Direitos} ; \mathrm{BE}=\mathrm{Bem}$-Estar Emocional; $\mathrm{BF}=\mathrm{Bem}$ Estar Físico; BM=Bem-Estar Material; QV=Qualidade de Vida; ${ }^{* *} p<.001 ;{ }^{*} p<.05$. 


\subsection{Fiabilidade Teste-Reteste}

A EPR-CJ foi duplamente administrada a dois cuidadores das crianças e jovens com DI, pelo mesmo entrevistador, com um intervalo de 2 a 3 semanas após a sua primeira aplicação. Este período de administração é recomendado na literatura como adequado para minimizar a probabilidade dos participantes se lembrarem das respostas iniciais ou sofrerem alterações significativas que pudessem condicionar as respostas (DeVon et al., 2007). A fiabilidade do teste-reteste pode ser classificada como moderada $(.80>r<.89)$ ou alta $(r \geq .90$; Hartley \& MacLean, 2006). Neste estudo participaram 33 profissionais de educação que prestavam apoio a crianças e jovens com DI e 33 familiares. A idade do pessoal de apoio estava compreendida entre os 43 e os 57 anos $\left(M_{\text {idade }}=47.64, D P=4.16\right)$ e a idade dos familiares entre os 26 e os 52 anos $\left(M_{\text {idade }}=43.36, D P=7.30\right)$.

A Tabela 8 ilustra as correlações de Pearson para quantificar a fiabilidade do teste-reteste, entre os profissionais e os familiares de crianças e jovens com DI. Observaram-se valores estatisticamente significativos entre as duas administrações da EPR-CJ, com um intervalo entre 2 a 3 semanas, demonstrando bons índices de replicabilidade e estabilidade. Todas as correlações de Pearson foram altas $(r$ entre $.70 \mathrm{e}$ .89) ou muito altas $(r \geq .90)$. O teste-reteste do Índice de QV dos profissionais foi alto $(r=.95)$, o mesmo sucedendo com o Índice de QV relatado pelos familiares $(r=.90)$.

Tabela 8 Fiabilidade teste-reteste

\begin{tabular}{l|c|c}
\hline \multirow{2}{*}{ Domínios } & $\begin{array}{c}\text { Relato dos Cuidadores } \\
\text { (Profissionais) }\end{array}$ & $\begin{array}{c}\text { Relato dos } \\
\text { Cuidadores (Família) }\end{array}$ \\
\cline { 2 - 3 } & $r$ & $r$ \\
\hline Desenvolvimento Pessoal & $.97 * *$ & $.90^{* *}$ \\
\hline Autodeterminação & $.88^{* *}$ & $.74 * *$ \\
\hline Relações Interpessoais & $.93^{* *}$ & $.90^{* *}$ \\
\hline Inclusão Social & $.98^{* *}$ & $.92^{* *}$ \\
\hline Direitos & $.92^{* *}$ & $.97 * *$ \\
\hline Bem-Estar Emocional & $.78^{* *}$ & $.98^{* *}$ \\
\hline Bem-Estar Físico & $.97 * *$ & $.70^{* *}$ \\
\hline Bem-Estar Material & $.97 * *$ & $.91 * *$ \\
\hline Índice de QV & $.95 * *$ & $.90^{* *}$ \\
\hline
\end{tabular}

Nota: $r=$ Correlação de Pearson; **p<.001. 


\subsection{Consistência Interna}

A consistência interna da escala e das suas dimensões foi verificada através do alfa de Cronbach. O alfa de Cronbach acima de .80 é considerado excelente, entre .70 e .80 é adequado e abaixo de .70 é inadequado (Andresen, 2000; Li et al., 2013).

Na Tabela 9 são apresentados os valores do alfa de Cronbach das duas partes da EPR-CJ. No autorrelato, os valores de consistência interna dos oito domínios de QV variaram entre os domínios das Relações Interpessoais $(\alpha=.41)$ e da Autodeterminação $(\alpha=.70)$. Na segunda parte da escala, os valores oscilaram entre os domínios do Bem-Estar Físico $(\alpha=.54)$ e do Bem-Estar Emocional $(\alpha=.79)$. A consistência interna dos três fatores da escala foi adequada $(\alpha \geq 70)$. No autorrelato, o fator da Independência apresentou o valor mais elevado $(\alpha=.78)$, enquanto no relato dos cuidadores o fator da Participação Social mostrou uma consistência interna excelente $(\alpha=.82)$. O Índice de QV do relato dos cuidadores $(\alpha=.87)$ foi mais elevado, comparativamente ao Índice de QV autorrelatado pelas crianças e jovens com DI $(\alpha=.81)$. Não obstante, os Índices de QV da EPR-CJ confirmaram a elevada consistência interna do instrumento, tanto nos participantes com DI como nos profissionais de educação.

Tabela 9 Consistência interna dos domínios da Escala Pessoal de Resultados-Crianças e Jovens

\begin{tabular}{l|c|c}
\hline & Autorrelato & Relato dos Cuidadores \\
\hline Independência & .78 & .73 \\
\hline Desenvolvimento Pessoal & .68 & .74 \\
\hline Autodeterminação & .70 & .74 \\
\hline Participação Social & .73 & .82 \\
\hline Relações Interpessoais & .41 & .68 \\
\hline Inclusão Social & .66 & .71 \\
\hline Direitos & .52 & .66 \\
\hline Bem-Estar & .73 & .78 \\
\hline Bem-Estar Emocional & .62 & .79 \\
\hline Bem-Estar Físico & .61 & .54 \\
\hline Bem-Estar Material & .62 & .76 \\
\hline Índice de QV & .81 & .87 \\
\hline
\end{tabular}




\subsection{Acordo Entre Observadores}

O acordo entre observadores foi examinado através das correlações de Pearson, possibilitando avaliar a concordância entre diferentes inquiridos, de uma forma independente (Andresen, 2000), bem como a relação entre o autorrelato e o relato dos cuidadores (Simões et al., 2015b). O acordo entre observadores foi avaliado de acordo com os seguintes critérios: pobre $<.40$, moderado a bom $\geq .40<.75$ e excelente $\geq .75$ (Andresen, 2000; Li et al., 2013). Nesta investigação, para cada criança e jovem com DI que foi avaliada no autorrelato $\left(n=54, M_{\text {idade }}=12.48, D P=2.93\right.$ ), foi preenchido de forma independente o relato dos cuidadores por um profissional de educação $\left(n=54, M_{\text {idade }}=46.59, D P=5.68\right)$.

Na Tabela 10 são apresentados os valores do acordo entre observadores, ou seja, a relação existente entre a avaliação da QV efetuada pelas crianças e jovens com DI e os profissionais de educação. Os valores variaram entre um acordo moderado no domínio das Relações Interpessoais $(r=.47)$, a um acordo excelente no domínio do Desenvolvimento Pessoal $(r=.81)$. Concomitantemente, existe um bom acordo na avaliação do Índice de QV entre o autorrelato e o relato dos cuidadores $(r=.65)$.

Tabela 10 Acordo entre observadores (crianças e jovens versus profissionais)

\begin{tabular}{l|c|c}
\hline & $r$ & $95 \%$ IC \\
\hline Desenvolvimento Pessoal & $.81 * *$ & {$[.69-.89]$} \\
\hline Autodeterminação & $.49 * *$ & {$[.26-.67]$} \\
\hline Relações Interpessoais & $.47 * *$ & {$[.23-.66]$} \\
\hline Inclusão Social & $.54 * *$ & {$[.32-.71]$} \\
\hline Direitos & $.51 * *$ & {$[.28-.68]$} \\
\hline Bem-Estar Emocional & $.59 * *$ & {$[.38-.74]$} \\
\hline Bem-Estar Físico & $.60 * *$ & {$[.40-.75]$} \\
\hline Bem-Estar Material & $.77 * *$ & {$[.63-.86]$} \\
\hline Índice de QV & $.65 * *$ & {$[.46-.78]$} \\
\hline Nota: $r=$ Correlação de Pearson; IC $=$ Intervalo de Confiança; $* * p<.001$.
\end{tabular}




\section{DISCUSSÃO E CONCLUSÃO}

$\mathrm{Na}$ presente investigação foi efetuada a adaptação cultural e examinada a validade e a fiabilidade da ECR-CJ. A validade e a fiabilidade são condições necessárias para a implementação desta escala, em Portugal, com base nos princípios de avaliação da QV que possibilitam conhecer os resultados pessoais (Claes, Van Hove, Van Loon, Vandevelde, \& Schalock, 2010b).

A fase da adaptação cultural respeitou as etapas especificadas na literatura (Simões \& Santos, 2014), tendo a comparação dos produtos resultantes dos tradutores originado a versão alvo do estudo da validade e fiabilidade.

O segundo objetivo desta investigação pretendeu examinar a validade da EPR-CJ, através da validade de conteúdo e da validade de constructo. A validade de conteúdo baseou-se no acordo e apreciação de 10 especialistas, com evidências empíricas, sobre a correspondência dos 48 itens e respetivos oito domínios de QV. O IVC dos itens deve ser igual ou superior a 78 quando há 6 ou mais especialistas envolvidos (Polit \& Beck, 2006) e é necessário, caso se aplique, remover os itens abaixo de .75 (Yaghmaie, 2003). Com a validade de conteúdo da EPR-CJ, a nossa investigação evidenciou que a proporção de acordo entre os 10 especialistas que participaram neste estudo apresentou resultados elevados (variou entre .95 e 1.00), não se tendo observado valores abaixo de .75 .

Quanto à relevância, clareza, simplicidade e ambiguidade dos itens que constituem a escala, observaram-se valores acima de .78, em todos os parâmetros em análise, comprovando que os 10 especialistas assumiram a pertinência das quarenta e oito perguntas de cada parte da EPR-CJ. O acordo universal do IVC confirmou, igualmente, a validade de conteúdo do instrumento, bem como a média do IVC da EPR-CJ (Polit \& Beck, 2006). Os coeficientes do Kappa de Cohen também se encontravam dentro dos valores recomendados na literatura (Watkins \& Pacheco, 2000; Wynd et al., 2003), contribuindo para a confiabilidade da validade de conteúdo do instrumento em análise.

Ainda relativamente ao segundo objetivo desta investigação, a validade de constructo do instrumento foi verificada através das 
correlações de Pearson (Hartley \& MacLean, 2006; Pestana \& Gageiro, 2005). Tanto no autorrelato, como no relato dos cuidadores observou-se pelo menos uma correlação moderada entre os domínios de QV, sendo que as correlações entre o Índice de QV e os oito domínios variaram entre valores moderados a altos, em ambas as secções. Deste modo, os dados revelaram que a validade de constructo da EPR-CJ é alta (Hartley \& MacLean, 2006).

O terceiro objetivo pretendeu analisar a fiabilidade da EPR-CJ, com base no teste-reteste, na consistência interna e no acordo entre observadores. Os resultados evidenciaram fiabilidade com base no testereteste, que foi efetuado com um intervalo entre 2 a 3 semanas. As correlações de Pearson foram altas ( $r$ entre .70 e .89) ou muito altas ( $r \geq .90$; Pestana \& Gageiro, 2005). Com base nos valores normativos (Hartley \& MacLean, 2006), o teste-reteste do Índice de QV relatado pelos profissionais foi alto $(r=.95)$, o mesmo sucedendo com o Índice de QV relatado pelos familiares $(r=.90)$.

A fiabilidade foi adequada, atendendo aos coeficientes da consistência interna (Andresen, 2000; Li et al., 2013). Os valores do alfa de Cronbach da primeira parte da EPR-CJ variaram entre os domínios das Relações Interpessoais $(\alpha=.41)$ e da Autodeterminação $(\alpha=.70)$. A consistência interna do autorrelato, aplicado na Bélgica, variou de .37 (Autodeterminação e Bem-Estar Físico) a .70 (Relações Interpessoais; Claes et al., 2015). Na perspetiva dos profissionais de educação, os valores oscilaram entre os domínios do Bem-Estar Físico $(\alpha=.54)$ e do Bem-Estar Emocional $(\alpha=.79)$, que foram congruentes com os resultados encontrados no estudo precedente ( $\alpha=.23$ no Bem-Estar Físico e $\alpha=.76$ no Bem-Estar Emocional; Claes et al., 2015).

A consistência interna dos três fatores da escala (Independência, Participação Social e Bem-Estar) foi adequada ( $\alpha \geq .70$; Andresen, 2000; Li et al., 2013). Os Índices de QV da EPR-CJ confirmam a elevada consistência interna do instrumento, tanto nos participantes com DI $(\alpha=.81)$ como nos profissionais de educação $(\alpha=.87)$. Os valores portugueses são semelhantes com a versão original (Claes et al., 2015), tendo sido observado no autorrelato .82 e no relato dos cuidadores .88. O Índice de $\mathrm{QV}$ do relato dos cuidadores foi mais elevado, comparativamente ao Índice de QV autorrelatado pelas crianças e jovens 
com DI, o que foi congruente com a investigação nacional com os adultos com DI (Simões et al., 2015b) e com os valores observados na Bélgica (Claes et al., 2015).

Relativamente ao acordo entre observadores, os valores variaram entre um acordo moderado no domínio das Relações Interpessoais ( $r=.47)$, a um acordo excelente no domínio do Desenvolvimento Pessoal ( $r=.81)$, sendo congruentes com os critérios definidos na literatura (Andresen, 2000; Li et al., 2013). De acordo com os mesmos autores, existe um bom acordo entre a avaliação da QV efetuada pelas crianças e jovens com DI e os profissionais de educação ( $r=.65$ ), ou seja, entre a QV medida por medidas autorrelatadas e as informações prestadas pelos cuidadores.

Em síntese, a investigação inicial sobre as propriedades psicométricas da EPR-CJ possibilitou concluir que a mesma irá manter uma estrutura semelhante ao instrumento inicialmente criado na Bélgica. Os coeficientes de validade e de fiabilidade suportaram a adequação desta escala para avaliar a QV das crianças e dos jovens portugueses com DI.

A EPR-CJ será fundamental para as práticas dos docentes de Educação Especial, no sentido que possibilita repensar a intervenção educativa, dentro de uma escola inclusiva, cuja principal meta pressupõe a melhoria da QV desta população. A inclusão pressupõe que todos os alunos, independentemente das dificuldades que apresentam, possam ter oportunidades para aceder ao currículo, com apoios adequados e participar com os seus pares nas escolas do ensino regular (Nilholm \& Göransson, 2017; Rodrigues \& Nogueira, 2010), pois “estar incluído é muito mais do que uma presença física: é um sentimento e uma prática de pertença entre a escola e a criança, isto é, o jovem sentir que pertence à escola e a escola sentir que é responsável por ele" (Rodrigues, 2003, p. 95).

A Convenção sobre os Direitos das Pessoas com Deficiência (CDPD), no artigo $24 .^{\circ}$, reforça a educação inclusiva, sublinhando que as crianças e os jovens com DI não podem ser excluídos do sistema geral de ensino (Nações Unidas, 2007). Salienta-se que o modelo concetual da QV possibilita medir, com validade e fiabilidade, os direitos garantidos (Karr, 2011) e recolher informações para monitorizar a implementação da CDPD, nomeadamente de trinta e quatro artigos (Verdugo, Navas, Gómez, \& Schalock, 2012). A EPR-CJ é crucial para monitorizar a 
implementação dos direitos dos alunos com DI, estimulando a inclusão de todos.

Pretende-se que esta ferramenta auxilie os apoios a prestar nos contextos inclusivos, criando um projeto de vida personalizado que aglutina uma intervenção transversal e holística dos diferentes intervenientes. Deste modo, a EPR-CJ possibilita avaliar e desenvolver as múltiplas facetas da vida das crianças e dos jovens, privilegiando-se o desenvolvimento da sua independência, participação social e bem-estar, com especial enfoque no desenvolvimento da autodeterminação e do exercício dos direitos.

Não obstante, o tamanho da amostra e alguns resultados de consistência interna das dimensões da escala apontam a necessidade de novos estudos psicométricos. Assim, em investigações futuras torna-se necessário alargar o número de indivíduos da amostra, com o intuito de se realizar novas análises estatísticas relativas à validade e à fiabilidade, salientando-se a análise fatorial confirmatória da escala.

\section{FONTES E BIBLIOGRAFIA}

American Psychiatric Association (APA). (2014). Diagnostic and statistical manual of mental disorders (5th ed.). Washington, DC: American Psychiatric Association.

Andresen, E. (2000). Criteria for assessing the tools of disability outcomes research. Archives of Physical Medicine and Rehabilitation, 81(2), S15-S20. doi:10.1053/apmr.2000.20619

Araújo, J., Dourado, M., \& Ferreira, P. (2015). Instrumentos de medição da qualidade de vida em idade pediátrica em cuidados paliativos. Acta Médica Portuguesa, 28, 501-512.

Brown, I. (2017). Quality of life: Challenges to research, practice and policy. Journal of Policy and Practice in Intellectual Disabilities, 14(1), 7-14. doi:10.1111/jppi.12185

Buntinx, W., \& Schalock, R. (2010). Models of disability, quality of life, and individualized supports: Implications for professional practice in intellectual disability. Journal of Policy and Practice in Intellectual Disabilities, 7(4), 283-294. doi:10.1111/j.1741-1130.2010.00278.x 
Claes, C., Mostert, R., Moonen, L., Van Loon, J., \& Schalock, R. (2015). Personal Outcomes Scale for Children and Adolescents: Instrument for the assessment of individual quality of life of children and adolescents between the ages of 6-18. Ghent, Belgium: Stichting Arduin.

Claes, C., Van Hove, G., Vandevelde, S., Van Loon, J., \& Schalock, R. (2010a). Person-centered planning: An analysis of its published literature and effectiveness. Intellectual and Developmental Disabilities, 48(6), 432-453. doi:10.1352/1934-9556-48.6.432

Claes, C., Van Hove, G., Van Loon, J., Vandevelde, S., \& Schalock, R. (2010b). Quality of life measurement in the field of intellectual disabilities: Eight principles for assessing quality of life-related personal outcomes. Social Indicators Research, 98(1), 61-72. doi:10.1007/s11205-009-9517-7

DeVon, H., Block, M., Moyle-Wright, P., Ernst, D., Hayden, S., Lazzara D., ... Kostas-Polston, E. (2007). A psychometric toolbox for testing validity and reliability. Journal of Nursing Scholarship, 39(2), 155164. doi:10.1111/j.1547-5069.2007.00161.x

Gaspar, T., \& Matos, M. (2008). Qualidade de vida em crianças $e$ adolescentes: Versão portuguesa dos instrumentos Kidscreen-52. Cruz Quebrada, Portugal: Aventura Social e Saúde.

Ghotra, S., McIsaac, J., Kirk, S., \& Kuhle, S. (2016). Validation of the 'Quality of Life in School' instrument in Canadian elementary school students. PeerJ, 4, e1567. doi:10.7717/peerj.1567

Gómez, L., Verdugo, M., Arias, B., \& Arias, V. (2011). A comparison of alternative models of individual quality of life for social service recipients. Social Indicators Research, 101(1), 109-126. doi:10.1007/s11205-010-9639-y

Hartley, S., \& MacLean, W. (2006). A review of the reliability and validity of Likert-type scales for people with intellectual disability. Journal of Intellectual Disability Research, 50(11), 813-827. doi:10.1111/j.1365-2788.2006.00844.X

Huang, C., Wang, T., Tang, F., Chen, I., \& Yu, S. (2017). Development and validation of a Quality of Life Scalefor elementary school students. International Journal of Clinical and Health Psychology, 17(2), 180-19. doi:10.1016/j.ijchp.2017.01.001 
Karr, V. (2011). A life of quality: Informing the UN Convention on the Rights of Persons with Disabilities. Journal of Disability Policy Studies, 22(2), 66-82.

Li, C., Tsoi, E., Zhang, A., Chen, S., \& Wang, C. (2013). Psychometric properties of self-reported quality of life measures for people with intellectual disabilities: A systematic review. Journal of Developmental and Physical Disabilities, 25(2), 253-270. doi:10.1007/s10882-012-9297-x

Luckasson, R., Borthwick-Duffy, S., Buntinx, W., Coulter, D., Craig, E., Reeve, A., ... Tassé, M. (2002). Mental retardation: Definition, classification, and systems of supports (10th ed.). Washington, DC: American Association on Mental Retardation.

Lombardi, M., Croce, L., Claes, C., Vandevelde, S., \& Schalock, R. (2016). Factors predicting quality of life for people with intellectual disability: Results from the ANFFAS study in Italy. Journal of Intellectual \& Developmental Disability, 41(4), 338-347. doi.org/10.3109/13668250.2016.1223281

Morato, P., \& Santos, S. (2007). Dificuldades intelectuais e desenvolvimentais: A mudança de paradigma na conceção da deficiência mental. Revista de Educação Especial e Reabilitação, 14(1), 51-55.

Nações Unidas. (2007). Convenção sobre os direitos das pessoas com deficiência. Lisboa, Portugal: Instituto Nacional para a Reabilitação. Disponível em http://www.inr.pt/content/1/50/organizacao-dasnacoes-unidas, consultado em 07/07/2018.

Nilholm, C., \& K., Göransson (2017). "What is meant by inclusion? An analysis of European and North American journal articles with high impact." European Journal of Special Needs Education, 32(3), 437451. doi:10.1080/08856257.2017.1295638.

Oliveira, O., Ribeiro, C., Simões, C., \& Pereira, P. (2018). Quality of life of children and adolescents with visual impairment. British Journal of Visual Impairment, 36(1), 42-56.

Pestana, M., \& Gageiro, J. (2005). Análise de dados para ciências sociais: A complementaridade do SPSS. Lisboa, Portugal: Edições Sílabo.

Polit, D., \& Beck, C. (2006). The content validity index: Are you sure you know what's being reported? Critique and recommendations. 
Research in Nursing \& Health, 29(5), 489-497. doi:10.1002/nur.20147

Rodrigues, D. (2003). Educação inclusiva: As boas e as más notícias. In D. Rodrigues (Org.), Perspectivas sobre a inclusão da educação à sociedade (pp. 89-101). Porto: Porto Editora.

Rodrigues, D., \& Nogueira, J. (2010). Educação especial e inclusiva em Portugal: Factos e opções. Revista Educación Inclusiva, 3(1), 97-109. Schalock, R., Brown, I., Brown, R., Cummins, R., Felce, D., Matikka, ... Parmenter, T. (2002). Conceptualization, measurement and application of quality of life for persons with intellectual disabilities: Report of an international panel of experts. Mental Retardation, 40(6), 457-470.

doi:10.1352/0047-6765(2002)040<0457:CMAAOQ>2.0.CO;2

Schalock, R., Buntinx, W., Borthwick-Duffy, S., Luckasson, R., Snell, M., Tassé, M., \& Wehmeyer, M. (2007). User's guide-mental retardation: Definition, classification and systems of supports (10th ed.). Washington, DC: American Association on Intellectual Disability.

Schalock, R., Gardner, J., \& Bradley, V. (2007). Quality of life for people with intellectual and other developmental disabilities: Applications across individuals, organizations, communities, and systems. Washington, DC: American Association on Intellectual Disability.

Schalock, R., Luckasson, R., Tassé, M., \& Verdugo, M. (2018). A holistic theoretical approach to intellectual disability: Going beyond the four current perspectives. Intellectual and Developmental Disabilities, 56(2), 79-89. doi:10.1352/1934-9556-56.2.79

Schalock, R., \& Verdugo, M. (2002). Handbook on quality of life for human service practitioners. Washington, DC: American Association on Mental Retardation.

Schalock, R., Verdugo, M., \& Gómez, L. (2011). Evidence-based practices in the field of intellectual and developmental disabilities: An international consensus approach. Evaluation and Program Planning, 34(3), 273-282. doi:10.1016/j.evalprogplan.2010.10.004

Schalock, R., Verdugo, M., Gómez, L., \& Reinders, H. (2016). Moving us toward a theory of individual quality of life. American Journal on 
Intellectual and Developmental Disabilities, 121(1), 1-12. doi:10.1352/1944-7558-121.1.1

Simões, C., \& Santos, S. (2014). Cross-cultural adaptation, validity and reliability of the Escala Pessoal de Resultados. Social Indicators Research, 119(2), 1065-1077. doi:10.1007/s11205-013-0515-4

Simões, C., \& Santos, S. (2017). The impact of personal and environmental characteristics on quality of life of people with intellectual disability. Applied Research in Quality of Life, 12(2), 389408. doi:10.1007/s11482-016-9466-7

Simões, C., \& Santos, S. (2018). Qualidade de vida, comportamento adaptativo e apoios: Compreender a relação entre constructos na dificuldade intelectual e desenvolvimental. Cruz Quebrada: Edições Faculdade de Motricidade Humana.

Simões, C., Santos, S., \& Biscaia, R. (2016). Validation of the Portuguese version of the Personal Outcomes Scale. International Journal of Clinical and Health Psychology, 16(2), 186-200. doi:10.1016/j.ijchp.2015.11.002

Simões, C., Santos, S., Biscaia, R., \& Thompson, J. (2016). Understanding the relationship between quality of life, adaptive behavior and support needs. Journal of Developmental and Physical Disabilities, 28, 849-870. doi:10.1007/s10882-016-9514-0

Simões, C., Santos, S., \& Claes, C. (2015a). Quality of life assessment in intellectual disabilities: The Escala Pessoal de Resultados versus the World Health Quality of Life-BREF. Research in Developmental Disabilities, 37(2), 171-181. doi:10.1016/j.ridd.2014.11.010

Simões, C., Santos, S., \& Claes, C. (2015b). The Portuguese version of Personal Outcomes Scale: A psychometric validity and reliability study. Intellectual and Developmental Disabilities, 53(2), 129-142. doi:10.1352/1934-9556-53.2.129

Simões, C., Santos, S., Claes, C., Van Loon, J., \& Schalock, R. (2017). Avaliação da qualidade de vida na dificuldade intelectual $e$ desenvolvimental: Manual de administração da Escala Pessoal de Resultados. Coimbra: FORMEM.

Verdugo, M., Navas, P., Gómez, L., \& Schalock, R. (2012). The concept of quality of life and its role in enhancing human rights in the field of 
intellectual disability. Journal of Intellectual Disability Research, 56(11), 1036-1045. doi:10.1111/j.1365-2788.2012.01585.x

Watkins, M., \& Pacheco, M. (2000). Interobserver agreement in behavioral research: Importance and calculation. Journal of Behavioral Education, 10(4), 205-212. doi:10530819/00/12000205\$18.00/0

World Medical Association. (2008). Declaration of Helsinki: Ethical principles for medical research involving human subjects. Disponível em http://www.bioetica.ufrgs.br/helsin7.pdf, consultado em 26/04/2012.

Wynd, C., Schmidt, B., \& Schaefer, M. (2003). Two quantitative approaches for estimating content validity. Western Journal of Nursing Research, 25(5), 508-518. doi:10.1177/0193945903252998

Yaghmaie, F. (2003). Content validity and its estimation. Journal of Medical Education Spring, 3(1), 25-27. 